\title{
Optimal Airflow Performance for FOUP Systems in Cleanrooms Using SVE Quantification Method
}

\author{
Yoshihide Suwa ${ }^{1}$, Jin Chul Park ${ }^{2}$ and Yong-Shik Kim ${ }^{3}$ \\ ${ }^{1}$ Visiting Professor, Department of Mechanical and Environmental Informatics, \\ Graduate School of Information Science and Engineering, Tokyo Institute of Technology, Japan \\ ${ }^{2}$ Associate Professor, School of Architecture, Chung-Ang University, Korea \\ ${ }^{3}$ Associate Professor, Department of Architectural Engineering, University of Incheon, Korea
}

\begin{abstract}
This research examined the possibility of optimizing airflow performance of cleanrooms with FOUP (Front Opening Unified Pod) systems used in $300 \mathrm{~mm}$ wafer lines for VLSI production. Numerical simulations using computational fluid dynamics (CFD) were carried out to obtain airflow states in cleanrooms with various design conditions. Each result was evaluated using a new quantification method and the cleanroom performance was then compared to it. The proposed method was based on the idea of Scale for Ventilation Efficiency (SVE), which is used to measure building ventilation. It enabled quantitative evaluation of the airflow performance independent from other factors and could be used to help design optimal cleanrooms. The study found that cleanroom performance changes with varying supply types and filter coverage, and that there is a proper combination of these parameters. Therefore, it is possible to develop the most efficient FOUP cleanroom system using this method.
\end{abstract}

Keywords: cleanroom; airflow performance; CFD techniques; FOUP system; SVE; 300mm wafer lines

\section{Introduction}

Recently, a production of VLSIs using 300mm wafer lines was started with many of these production lines employing FOUP ${ }^{1)}$ systems.

Although the detail specifications of FOUP systems have been standardized by committees such as SEMATEC I300I, a clear plan still does not exist for cleanrooms in which the system should be stored. A new design concept, which can be adapted for FOUP cleanrooms, is required for a full-scale shift to $300 \mathrm{~mm}$ wafer lines.

In this research work, a quantification technique to measure airflow performance was devised to systematically examine the optimal design of cleanrooms utilizing FOUP systems. This paper outlines this method and describes the results of the study.

*Contact Author: Jin Chul Park, Associate Professor,

School of Architecture, Chung-Ang University, 221 Heukseok-

Dong, Dongjak-Ku, Seoul, 156-756, Republic of Korea

Tel: +82-11-354-4843 Fax: +82-2-816-5261

E-mail: jincpark@cau.ac.kr

(Received April 8, 2010 ; accepted September 2, 2010)

\section{Evaluation Method for Cleanroom Airflow Performance}

\subsection{Issues with cleanroom design}

Until now, most cleanroom requirements have been put forth in the "Cleanliness Classes" found in ISO14644- ${ }^{2)}$, Fed.Std.209E ${ }^{3)}$ etc., and designers have tried to satisfy these performance specifications. However, the "Cleanliness Class" is not concerned with a cleanroom's airflow performance per se. The "Cleanliness Class" is based on particle concentration. The value can be found as a result of the balance of contaminant removal and the amount of contaminant generation. Moreover, in the planning stages of developing new cleanrooms, detailed amounts of contaminant generation cannot be obtained. For this reason, designers should consider the purpose of the cleanroom and perform the concrete design experimentally.

On the other hand, numerous studies have been conducted on the relationship between airflow state and contaminant distribution using experiments and CFD simulations. In most of these studies however, researchers assumed limited situations. Although the qualitative phenomenon analysis is a possible analysis for 3-dimensional airflow or contaminant distribution, it is difficult to perform a quantitative examination of how cleanroom performance changes according to design conditions. 


\subsection{Age-of-air and SVE}

The authors thought that most of these problems originated in not having a quantitative evaluation method for airflow performance itself. If the airflow performance in cleanrooms is evaluated independently from the amount of contaminant generation, the relationship between design conditions and cleanroom performance can be generalized and associated, thereby enabling examination of the optimal design conditions attained. This also leads to a low cost cleanroom design.

In this study, an evaluation method for cleanroom performance is proposed ${ }^{4), 5}$. This method is based on the ideas of "age-of-air" 6) and "SVE"1), both of which are used to measure building ventilation. "Age-of-air" is defined as the elapsed time passed after a unit of air is supplied to an arbitrary position. A zone where "age-of-air" shows a large value has less ventilation performance, and therefore a higher possibility of contamination. A zone with a small "age-of-air" value has good ventilation performance. On the other hand, SVEs are the indices carried out from Fourier series low-order-components for 3-dimensional concentration distributions. Indices SVE-1 to SVE-6 shown in Table 1. were proposed by Murakami and Kato ${ }^{7), 8}$. Among these indices, SVE-3 agrees with normalized "age-ofair" and normalized contaminant concentration when the uniform source of contaminant is assumed.

Table 1. Indices SVE-1 to SVE-6

\begin{tabular}{c|l}
\hline SVE-1 & $\begin{array}{l}\text { Indoor mean concentration when arbitrary } \\
\text { generation sources are assumed }\end{array}$ \\
\hline SVE-2 & $\begin{array}{l}\text { The radius of the concentration rule region from } \\
\text { arbitrary generation sources }\end{array}$ \\
\hline SVE-3 & $\begin{array}{l}\text { Normalized age of air or normalized concentration } \\
\text { when the uniform source is assumed }\end{array}$ \\
\hline SVE-4 & The sphere of influence of each supply \\
\hline SVE-5 & The sphere of influence of each exhaust \\
\hline SVE-6 & Normalized residual lifetime of air \\
\hline
\end{tabular}

\subsection{Evaluation of cleanroom airflow performance using SVE}

Fundamentally, cleanrooms must remove contaminants from the room by supplying clean air through filters. If airflow stagnation occurs within the cleanroom, the SVE in that zone would show a high value, the contaminant concentration would become higher than in other zones and would occur more easily. If we observe the SVE levels in such cleanrooms, zones with the possibility of contamination can be seen. Fig.1. shows the SVE levels around installed equipment in a unidirectional airflow cleanroom ${ }^{4}$.

Fig.1. also illustrates that the SVE level increases in flow separation around the equipment and near the walls. These SVE levels coincide well with the fact that it is difficult to remove contaminants in these zones.

SVE-3 is advantageous in designing the airflow in cleanrooms. Consider the mean value of SVE-3 in target rooms (mean value of the SVE-3 is described

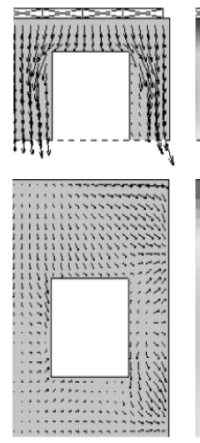

a) Airflow distrobution
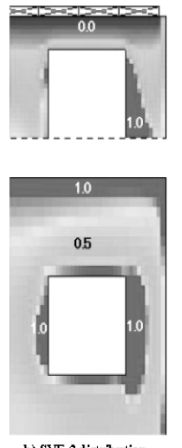

b) SYE-3 distrhution

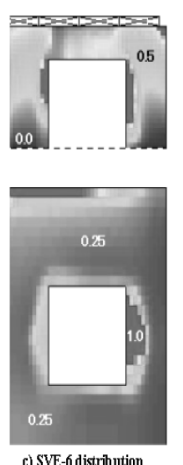

c) SVE-6 distribution
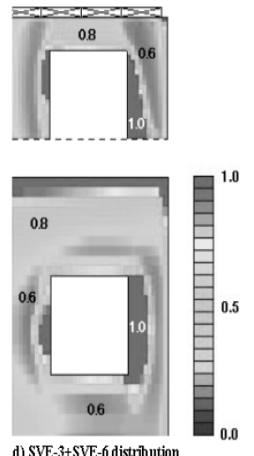

d) SYE-3+SYE-6 distrnbution
Fig.1. SVE Levels Around Installed Equipment in a Unidirectional Airflow Cleanroom

as $<$ SVE-3 $>$ in the following sections). Fig.2. shows typical indoor airflow states. The $<$ SVE- $3>$ in a completely mixed flow zone becomes 1.0 and the $<$ SVE-3 $>$ in an ideal piston flow zone becomes 0.5 . As shown in Fig.3., unidirectional airflow cleanrooms produce a downward airflow from a filtered ceiling towards a grated floor, making airflow in the room similar to that of the piston flow zone mentioned above.

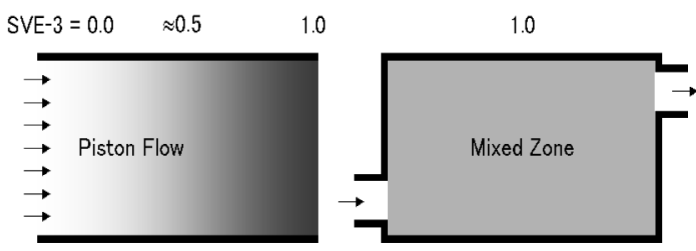

Fig.2. Typical Flow States and $<$ SVE-3 $>$

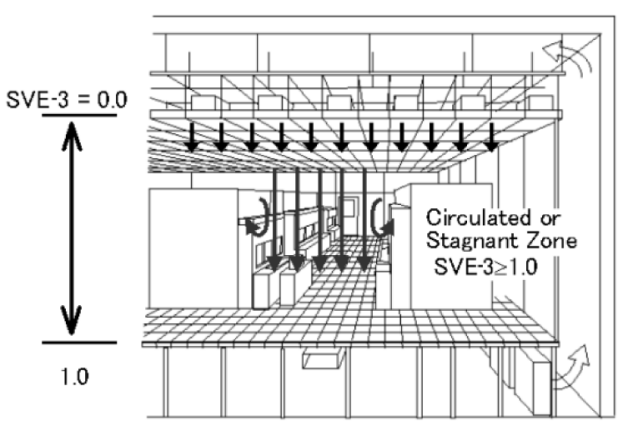

Fig.3. Similarity of Airflow in Piston Flow Domains and Unidirectional Airflow Cleanrooms

The $<$ SVE-3 $>$ in unidirectional airflow cleanrooms with the most desirable airflow state becomes 0.5 and increases according to stagnation or circulation. For this reason, the authors hypothesized that the airflow performance in unidirectional airflow cleanrooms could be evaluated quantitatively regarding how the $<$ SVE-3 $>$ exceeds 0.5 . To this point, the authors have evaluated the $<\mathrm{SVE}-3>$ in various cleanroom models. In many cases, the $<$ SVE- $3>$ of unidirectional airflow cleanrooms was found to lie between 0.5 and 1.0 , with the quality of cleanroom and the $<\mathrm{SVE}-3>$ 
showing a good correlation ${ }^{9)}$. Because this method is also applicable to cleanrooms with different design conditions, the authors have obtained a reasonable and advantageous method for highly complicated airflow characteristics involved in 3-dimensional flow distribution described with only one variable. Furthermore, airflow performance in unidirectional airflow cleanrooms can be studied by comparing this value.

\section{Optimizing Airflow Performance of FOUP Cleanrooms}

3.1 Selecting the model for analysis

Since the shift of semiconductor wafer size from $200 \mathrm{~mm}$ to $300 \mathrm{~mm}$ was implemented in 1994 , the standardization of manufactured equipment and machine-interfaces was completed through international committees such as SEMATECH I300I and J300. However, only the basic requirements of cleanroom design were shown in the guidelines published by $\mathrm{J} 300^{10)}$. Table 2 . shows the requirements for open cassette and FOUP cleanrooms using $300 \mathrm{~mm}$ wafer lines. There are no regulations for concrete air supply systems or the like.

Table 2. Requirements of $300 \mathrm{~mm}$ Wafer Line Cleanrooms

\begin{tabular}{|c|c|}
\hline $\begin{array}{l}\text { Cleanliness } \\
\text { Class }\end{array}$ & $\begin{array}{l}\text { [Open Cassette] } \\
\text { Process zones: class } 0.1 \sim \text { class } 1 \\
\text { Other zones: class } 1000 \sim \text { class } 10000 \\
\text { [Pod (FOUP)] } \\
\text { Inside pods: class } 0.1 \sim \text { class } 1 \\
\text { Inside tools: class } 0.1 \sim \text { class } 1 \\
\text { In Clean-rooms: class } 100 \sim \text { class } 1000\end{array}$ \\
\hline $\begin{array}{c}\text { Chemical } \\
\text { Contamination }\end{array}$ & - \\
\hline $\begin{array}{l}\text { Temperature } \\
\text { (DB) }\end{array}$ & $23 \pm 1^{\circ} \mathrm{C}$ \\
\hline Humidity (RH) & $35 \% \sim 55 \%$ \\
\hline
\end{tabular}

The authors assumed that the cleanroom models to be studied as FOUP cleanrooms must satisfy the following specifications.

(1) Reduce the number of installed filters and the running cost.

(2) For piping or an assistant machine installation space, a sub-fab grated floor should be prepared.

(3) Extreme localization should be avoided so that equipment layout can be changed easily, but a reasonable level of cleanliness should be achieved in the entire cleanroom.

In consideration of the basic requirements in Table 2., a cleanroom model was developed as shown in Fig.4. The test cleanroom is a bowl room type $(16 \mathrm{~m}(\mathrm{~W})$ by $22 \mathrm{~m}(\mathrm{~L})$ by $3.5 \mathrm{~m}(\mathrm{H})$ ), in which 8 sets of equipment are installed. Equipment size was determined by referring to currently available products. The height of the subfab space in each model was set to $4 \mathrm{~m}$. As mentioned above, the merit in adopting a mini-environmental system is to reduce the controlled space and running cost of the cleanroom. Here, the models have different filter coverage as shown in Fig.5. Some models have the spread type supply systems as shown in Fig.6. Characteristics of the spread type supply system are expressed in the parameter "FDR" as the filter's diffusion ratio. This ratio is defined using the ratio of horizontal and perpendicular airflow volume $(\mathrm{FDR}=$ [horizontal airflow volume]: [perpendicular airflow volume]). Airflow distribution and the $\langle\mathrm{SVE}-3\rangle$ in each model is simulated using the CFD technique, and the difference of airflow performances is studied.

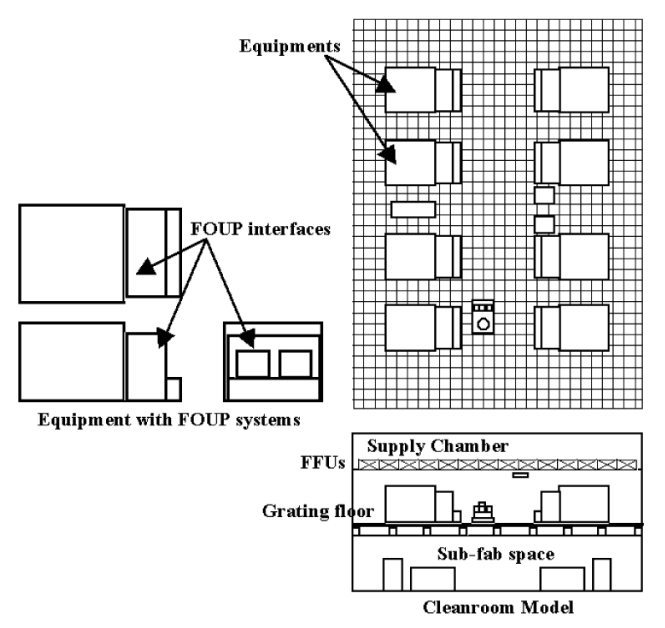

Fig.4. Test Cleanroom Model

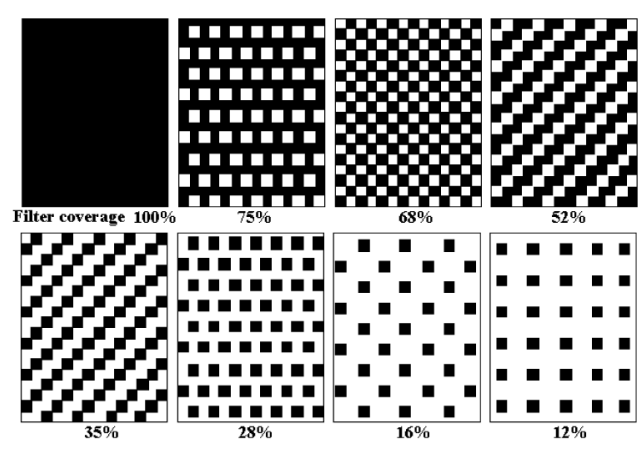

Fig.5. Layout of Tested Supply Air Filters

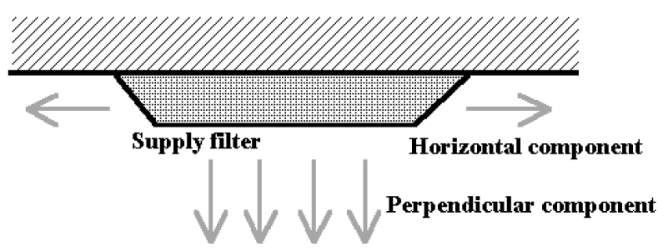

Fig.6. Spread Type Air Filter

\subsection{SVE-3 distribution}

SVE-3 distribution at $1.5 \mathrm{~m}$ above floor level [assuming filter coverage as $12 \%, 35 \%, 68 \%$, respectively, and FDR $=0: 10$ (only perpendicular supply flow)] are shown in Fig.7. In all cases, the influence of the filter layout is large, and the SVE-3 varies greatly. If the filter coverage is made smaller, the cleanliness 
level varies greatly. On the other hand, if the spread type supply system is adopted and the filter's diffusion ratio is adjusted, the cleanliness level in the cleanroom can be improved. Fig.8. shows the SVE-3 levels of the cleanroom with different filter diffusion ratios (filter coverage is fixed to $68 \%$ ). The results showed that the variation in the SVE-3 is reduced when horizontal airflow volume is increased. However, when the ratio of horizontal airflow volume is increased too much, the variation in the SVE-3 may become correspondingly larger. This means conditions such as filter layout and filter diffusion ratio should be selected with consideration to balance.
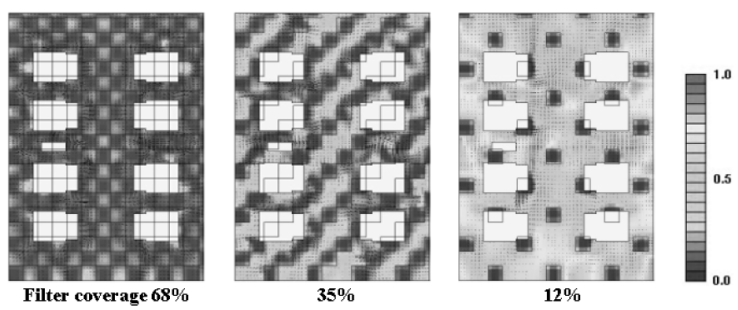

Fig.7. Difference in SVE-3 Distribution by Filter Coverage and Layout
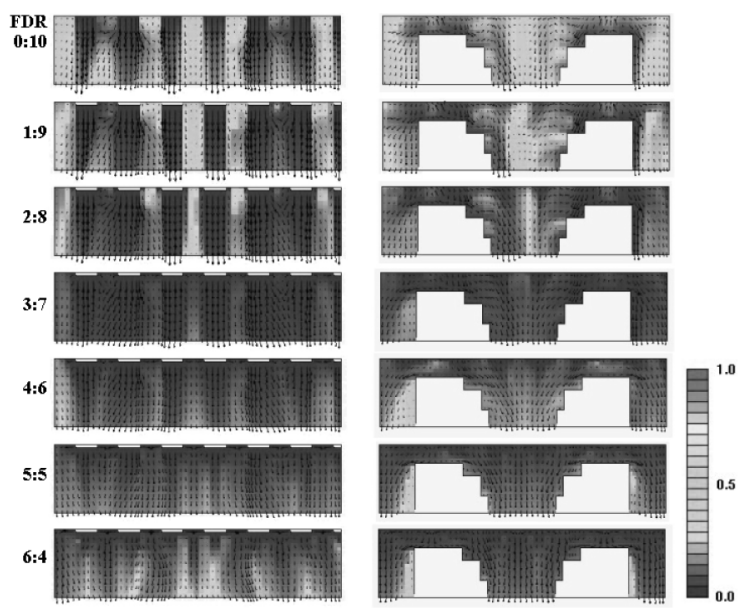

Fig.8. Difference in SVE-3 Distribution by Filter Diffusion Ratio

\subsection{Comparison of $<$ SVE-3 $>$}

The $<$ SVE- $3>$ of each model was calculated and the parameters compared. The results are shown in Figs.9. and 10. In Fig.9., the $<$ SVE-3 $>$ increases rapidly when filter coverage is less than $40 \%$. Research on nonunidirectional airflow cleanrooms by Takagi et al. ${ }^{11)}$ has concluded that the limit of filter curtailment is $30 \%$ in filter coverage.

Fig.9. also shows that the same threshold may also exist in unidirectional airflow cleanrooms. On the other hand, it was found that sufficient airflow may be acquired in the case where the spread type supply system is adopted, even if the filter coverage is low. This means the adoption of a spread type supply system is also advantageous for unidirectional airflow cleanrooms with reduced supply air filters.

Fig.10. shows the summarized results using the filter diffusion ratio as a parameter. Airflow performance is influenced by filter coverage in some cases. This tendency is different for each value of FDR. Therefore, the optimal filter layout and type should be chosen by comparing the $<$ SVE-3 $>$ levels with consideration to these relationships.

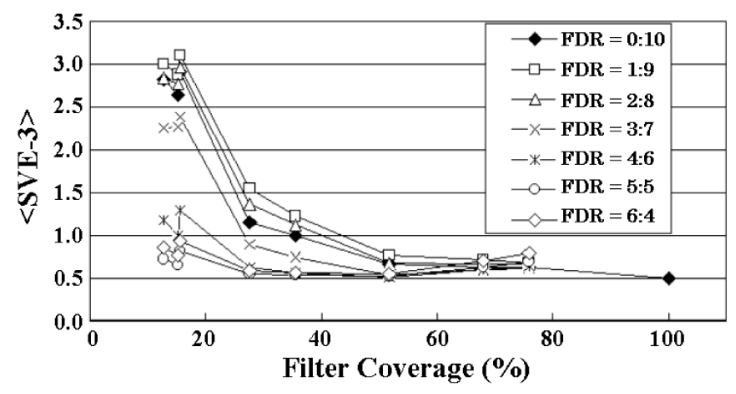

Fig.9. Relationship between the Filter Coverage and $<$ SVE-3 $>$

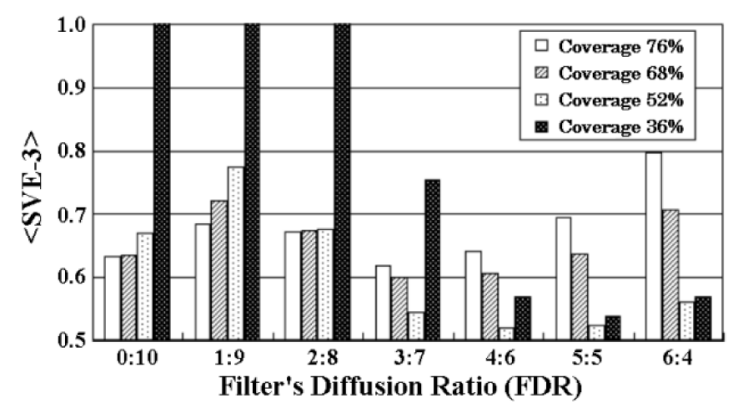

Fig.10. Relationship between the Filter's Diffusion Ratio Coverage and $<$ SVE-3 $>$

\section{Conclusion}

The airflow performance in various cleanroom models was evaluated for the purpose of studying the optimal design which can be adapted for FOUP cleanrooms. The airflow distribution of each model was simulated using the CFD technique and the performance was evaluated using the newly devised quantification method.

As a result of this study, it was found that cleanroom performance changes according to a proper combination of air supply types and air-filter coverage. The authors conclude that the adoption of a spread type air supply system was advantageous for FOUP cleanrooms with reduced supply air filters. Study of cleanroom performance will be continued to obtain concepts for future cleanroom systems.

\section{References}

1) P. Balentine, G. Gallagher (1995) Proc. of SEMI Technology Symposium, and http://www.infab.com/1330/Default.Htm

2) ISO-14644-1 (1999) Cleanrooms and Associated Controlled Environments - Part 1, Classification of Air Cleanliness.

3) Fed. Std. 209E (1992) Airborne Particulate Cleanliness in Cleanrooms and Clean Zones. 
4) Y. Suwa, K. Yosa, et al. (2000) A Study on the Evaluation Method for Cleanroom Airflow State - Part 1: A Study on the use of the Scale for Ventilation Efficiency (SVE) for Cleanroom Problems (in Japanese), $18^{\text {th }}$. Ann. Tech. Meeting on Air Cleaning and Contamination Control, Japan Air Cleaning Association, p.240.

5) T. Nagura, Y. Suwa, et al.(2000) A Study on the Evaluation Method for Cleanroom Airflow State - Part 2: An Application on the Scale for Ventilation Efficiency for Cleanroom Design (in Japanese), $18^{\text {th }}$. Ann. Tech. Meeting on Air Cleaning and Contamination Control, Japan Air Cleaning Association, p.243.

6) M. Sandbegg, M. Sjoeberg (1983) The Use of Moment for Assessing Air Quality in Ventilated Rooms, Building Environment 18, p. 181 .

7) S. Murakami, S. Kato (1986) New Scale for Evaluating Ventilation Efficiency (in Japanese), Transactions of the Society of Heating, Air-Conditioning and Sanitary Engineers of Japan, No. 32, pp.91102.

8) S. Kato, S. Murakami, et al. (1994) New Scale for Evaluating Ventilation Efficiency as Affected by Supply and Exhaust Openings based on Spatial Distribution of Contaminant, $12^{\text {th }}$ International Symposium on Contamination Control, ICCCS, p.341.

9) Y. Suwa, K. Yosa (2001) A Study on the Evaluation Method for Cleanroom Airflow State - Part 3: Parametric Studies on the Ventilation Efficiency in Several Types of Cleanrooms with Different Supply Types and Filter Arrangements (in Japanese), $19^{\text {th }}$. Ann. Tech. Meeting on Air Cleaning and Contamination Control, Japan Air Cleaning Association, p.96

10) J300 (1996) $2^{\text {nd }}$ Lecture, ICS Factory Design for $300 \mathrm{~mm}$ Wafer Line Standardizing Study.

11) N. Nishimura, K. Takagi, Y. Suwa (2001) Basic Study on Evaluation Method of Non-unidirectional Cleanroom Using Age of Room Air (in Japanese), $18^{\text {th }}$ Symposium on Aerosol Science \& Tech., Japan Association of Aerosol Science and Technology, p.59. 